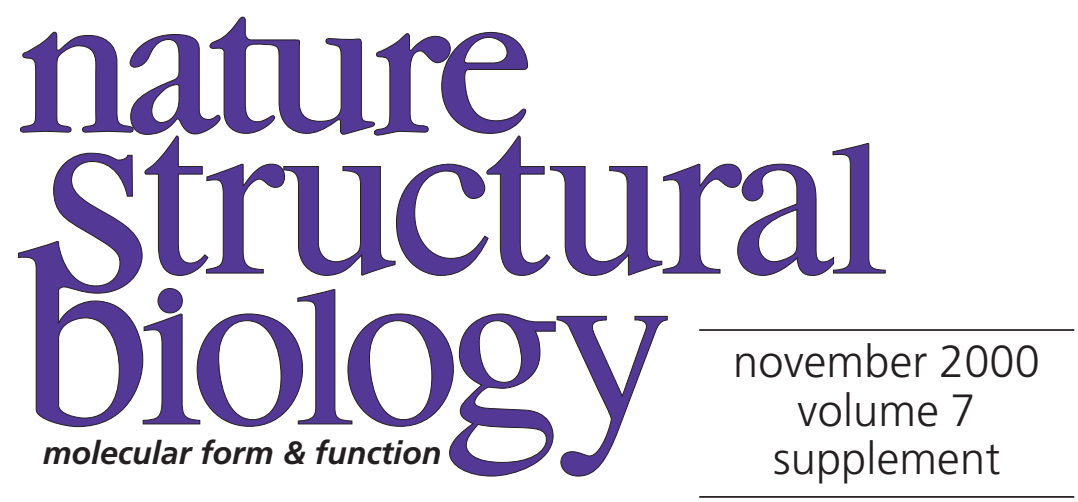

\section{A new era}

Welcome to the Structural Genomics supplement to Nature Structural Biology. We chose the topic of this special issue for two reasons. First, it is clear that structural biology is entering a new phase - that of high throughput, whole genome analysis - and this change warrants a closer look. Second, although the phrase 'Structural Genomics' has been in use for several years, many are still unsure of what it means exactly. We believe this supplement will go a long way to help this situation - to describe the current state and future plans of Structural Genomics efforts around the world, and to help delineate the possible benefits of this research.

We decided to present overviews of the field from many different viewpoints, as well as to delve more deeply into the details of the technical issues that underlie the research. To this end, the review articles in this supplement are divided into two sections. Broadly speaking, the articles in the Perspectives section attempt to answer the questions: Who? What? Why? and When? Those in the Progress section address the question: How? The reviews in the former section outline the goals of the field and the research groups involved, placing Structural Genomics solidly into the context of academic research, biotechnology, drug discovery, databases, and intellectual property. The latter set of articles should give the reader a better understanding of the practicalities - of how the goals put forward by the Structural Genomics field can be met, what major intellectual challenges remain, and what technical bottlenecks must be overcome to achieve high throughput structure determination, provide complete coverage of protein fold space, and accelerate general biological research by providing an extensive foundation of structural data.

Large sums of money have recently been allocated to Structural Genomics by several national funding initiatives, most notably in the United States and Japan. Many celebrate this move. But others meet it with apprehension, voicing concerns about the usefulness of moving away from the prevailing, and successful, model of hypothesis-driven research and about the impact on 'traditional' structural biology. This is not an unexpected reaction - after all, researchers in the field of Structural Genomics are embarking on a difficult and expensive enterprise, one that will likely be much more complicated and costly than the nearly complete project to sequence the human genome. The scientists who have contributed to this supplement are confident that their goals can and will be met, and that the wealth of new data that they will provide will revolutionize biology. The next decade will bear witness to these efforts, and their outcome.

We are pleased to acknowledge the financial support of SmithKline Beecham Pharmaceuticals and the US National Institute of General Medical Sciences in producing this supplement and distributing it to a wide audience. Their forewords, outlining their interests in Structural Genomics, can be found on pages 928 and 931. Of course, Nature Structural Biology carries the sole editorial responsibility for the remaining material — its content and rigorous peer review. We hope you enjoy these reviews and that they provide you with a more complete understanding of Structural Genomics. 